\title{
Deubiquitination in prostate cancer progression: role of USP22
}

\author{
Nivedita Nag${ }^{1}$, Samikshan Dutta² \\ 'Department of Microbiology, Sister Nibedita Government General Degree College for Girls, Kolkata 700027, India. \\ 2Department of Biochemistry and Molecular Biology, University of Nebraska Medical Center, Omaha, NE 68198-5870, USA.
}

Correspondence to: Dr. Samikshan Dutta, Department of Biochemistry and Molecular Biology, University of Nebraska Medical Center, BCC 6-12-391, 985870 Nebraska Medical Center, Omaha, NE 68198-5870, USA. E-mail: samikshan.dutta@unmc.edu

How to cite this article: Nag N, Dutta S. Deubiquitination in prostate cancer progression: role of USP22. J Cancer Metastasis Treat 2020;6:16. http://dx.doi.org/10.20517/2394-4722.2020.23

Received: 24 Mar 2020 First Decision: 14 Apr 2020 Revised: 7 May 2020 Accepted: 2 Jun 2020 Published: 18 Jun 2020

Science Editor: Zhou Wang Copy Editor: Cai-Hong Wang Production Editor: Tian Zhang

\begin{abstract}
Prostate cancer ( $\mathrm{PCa}$ ) is the leading cause of cancer death in men. With more therapeutic modalities available, the overall survival in PCa has increased significantly in recent years. Patients with relapses after advanced secondgeneration anti-androgen therapy however, often show poor disease prognosis. This group of patients often die from cancer-related complicacies. Multiple approaches have been taken to understand disease recurrence and to correlate the gene expression profile. In one such study, an 11-gene signature was identified to be associated with PCa recurrence and poor survival. Amongst them, a specific deubiquitinase called ubiquitin-specific peptidase 22 (USP22) was selectively and progressively overexpressed with PCa progression. Subsequently, it was shown to regulate androgen receptors and Myc, the two most important regulators of PCa progression. Furthermore, USP22 has been shown to be associated with the development of therapy resistant PCa. Inhibiting USP22 was also found to be therapeutically advantageous, especially in clinically challenging and advanced PCa. This review provides an update of USP22 related functions and challenges associated with PCa research and explains why targeting this axis is beneficial for PCa relapse cases.
\end{abstract}

Keywords: USP22, prostate cancer, SAGA, Deubiquitin

\section{INTRODUCTION}

Epidemiologically, prostate cancer ( $\mathrm{PCa}$ ) is the most common cancer in men and second most common cancer related death worldwide ${ }^{[1]}$. Over the past few years, treatment modalities have improved, albeit modestly, the overall survival of PCa patients. The fate of advanced PCa remains the same however, and androgen deprivation therapy (ADT) is the standard of care in such cases. PCa eventually recurs within

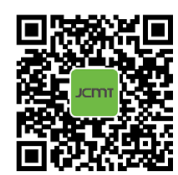


6 months to 2 years in the form of highly aggressive castration resistant prostate cancer (CRPC) ${ }^{[2-5]}$. While the treatment of CRPC with second generation ADT such as Abiraterone or Enzalutamide is promising, eventually, the cancer progresses to metastatic disease [called metastatic CRPC (mCRPC)], especially in the bone ${ }^{[6]}$. Chemotherapy with docetaxel is the first choice for treatment with mCRPC. Unfortunately, mCRPC patients have died due to complications related to metastatic transformation of $\mathrm{PCa}^{[7-11]}$. Interestingly, these mCRPC often lose the androgen-receptor dependency and are associated with the loss of tumor suppressor proteins such as tumor protein p53 (TP53) and retinoblastoma 1 (RB1 $)^{[12,13]}$. Genomewide sequencing analysis has found some of the unique variations in the chromosomal sequence but no such driver mutation/s in PCa has been ascertained to correlate with cancer progression ${ }^{[14-16]}$. Moreover, not all primary PCa cases progress to CRPC. Therefore, to understand the indolent vs. aggressive nature of PCa, gene-expression analysis is highly important. To correlate CRPC progression and to stratify the therapeutic regimen, high throughput sequencing analysis of various stages of PCa to correlate genetic expression profiles with the therapy-resistant state has been attempted. The expression of a 11-gene signature in primary prostate tumors was shown to correlate with therapeutic failure in PCa patients ${ }^{[17]}$. Further characterization has shown that this 11 -gene signature is a powerful predictor of distant metastasis and poor survival. Amongst these eleven genes, a specific deubiquitinases, named USP22, has been overexpressed following PCa progression. Further evidence indicates the importance of USP22 in a multi-faced pathway, which often correlates with a poor prognosis of PCa independently ${ }^{[18-20]}$.

\section{CLASSIFICATION OF DEUBIQUITINATING ENZYMES}

The protein ubiquitin ( $\mathrm{Ub}$ ) plays an important role in tissue homeostasis. Ub modification is a reversible phenomenon that is coordinated by the deubiquitination pathway ${ }^{[21-24]}$. Deubiquitinating enzymes (DUBs) belong to either cysteine proteases [such as ubiquitin-specific proteases (USPs), ubiquitin C-terminal hydrolases (UCHs), etc.] or metalloproteases [Jab1/Mov34/Mpr1 (JAMM)], which are important for maintaining normal physiological homeostasis ${ }^{[21,25]}$. Approximately 100 DUBs are encoded in the human genome. DUBs are involved in various physiological processes including the processing of Ub precursors, reversal of ubiquitination and removal of poly-ubiquitin chains ${ }^{[20,26]}$. Therefore, DUBs regulate a series of cellular processes and functions including proteolysis, apoptosis, cell cycle progression, gene expression, DNA repair, maintenance of telomeric length, spermatogenesis, and so on ${ }^{[27-30]}$. One such conserved ubiquitin-specific protease is USP22 and it has been well characterized, relating to various physiological and pathological processes ${ }^{[19,31]}$.

\section{UBIQUITIN-SPECIFIC PEPTIDASE 22}

The ubiquitin-specific peptidase 22 (USP22) belongs to the USPs family of DUBs and is highly conserved from yeast to vertebrates. In yeast, the USP22 homologue known as Ubp8, is complexed with Sgf73, Sgf11 and Sus1 to form the deubiquitylase module (DUBm) of the SAGA (Spt-Ada-Gen5 Acetyl transferase) complex. The SAGA complex has a multi-disciplinary role in gene-expression and RNA-transport. Like yeast Ubp8, USP22 also forms a DUBm complex with the human orthologue ATXN7L3, ENY2 and ATXN7 and functions as a DUB unit of the human SAGA complex ${ }^{[32,33]}$.

\section{ROLE OF USP22 IN CELLULAR PROCESSES}

As a part of the SAGA complex, transcriptional activation by deubiquitination of lysine-123 of histone$\mathrm{H} 2 \mathrm{~B}$ is enhanced ${ }^{[34,35]}$. Later it was identified that histone-H2A ubiquitination can be processed by USP 22 . Ubiquitination of $\mathrm{H} 2 \mathrm{~A}$ by the polycomb group of proteins is related to transcriptional repression. However, whether deubiquitination of $\mathrm{H} 2 \mathrm{Aub}$ (monoubiquitinated histone) by USP22 reverses the phenotype is not yet clearly established ${ }^{[36]}$.

Other than histones, USP22 also regulates the ubiquitination status of a large number of non-histone proteins. One of the most important functions of USP22 is to regulate telomeric length. Telomeric repeat 
binding factor 1 (TRF1) functions as a negative regulator of telomere length by inhibiting the access of telomerase to the telomeric region of chromosomes. Poly-ADP-ribosylation of TRF1 by Fbx4 releases it from the telomere, which in turn gets ubiquitinated and degraded by the proteasomal pathway. On the contrary, as a part of the SAGA complex, USP22, by deubiqitinating TRF1, restores its protein level and thereby maintains telomeric length. Depleting USP22 decreases TRF1 levels and enhances cell death by genotoxic insults ${ }^{[27]}$. The deubiquitination activity of USP22 is also important for the stability of Sirtuin 1 (SIRT 1$)^{[37]}$. By deacetylating, SIRT1 negatively regulates the transcriptional activity of p53 and thereby, p53 dependent apoptosis ${ }^{[38]}$. Deubiquitination also stabilizes another important transcription factor called c-Myc by the similar SIRT1 mediated pathway. In short, by deubiquitinating a number of transcriptional regulators such as Hes1, NFAT, COX-2, SNF1, etc., USP22 maintains their homeostatic functions within the cell ${ }^{[39-42]}$. A compensatory mechanism also involves SAGA, c-Myc and SIRT1. The enhanced stability of c-Myc through a USP22 dependent manner increases its transcriptional activity, which in turn, increases SIRT1 expression. However, the increase in SIRT1 levels enhances its deacetylation activity, which in turn, deacetylates USP22 and other SAGA components, thereby decreasing the enzymatic activity of USP22 ${ }^{[43]}$. Interestingly, not all deubiquitinations altered protein stability; it also changes the molecular function of the protein. Deubiquitinating lysine- 63 of FBP1 enhances its recruitment to the chromosome ${ }^{[44]}$. The role of USP22 in B cells is also important for its functionality. Complete ablation of USP22 in primary B cells was found to impair the classical non-homologous end joining and thereby, affects both $V(D) J$ recombination and class switch recombination for the development of various IgG and $\operatorname{IgE}$ subtypes ${ }^{[45]}$.

Little is known about USP22 regulation however. Reports indicate that USP22 transcription is regulated by Sp1 and the PKA/CREB dependent pathway ${ }^{[46,47]}$. USP22 is also regulated and stabilized by phosphorylation. Phosphorylation of USP22 at T147 and S237 by CDK1 increases the deubiquitination status of cyclin B1 in a cell cycle dependent manner. USP22 mediated deubiquitination of Cyclin B1 promotes its nuclear accumulation and cell cycle progression ${ }^{[48]}$. USP22 is ubiquitously expressed in human subjects as well as in mice. In mice, USP22 expression was detected as early as in E4.5. Loss of both USP22 alleles results in an embryonic lethality starting at E10.5 and no live embryos were recovered after E14.5. Embryonic expression patterns indicate that the potential functions of USP22 relate to the development of extra-embryonic tissues and the loss of function of embryonic USP22 fails to establish vascular interactions with the maternal circulatory system, which leads to immense hypoxic stress induced lethality. Loss of USP22 is also associated with impairments in transforming growth factor $\beta$, vascular endothelial growth factor receptor-2 and platelet derived growth factor signaling axes in endothelial cells, and pericytes have been shown to be implicated with detrimental effects on cell survival, differentiation and vessel formation. However, the heterozygous loss of USP22 in mice is still viable but with retardation of growth and brain development ${ }^{[3,45]}$. USP22 expression is also important for embryonic stem cell (ESC) differentiation into the embryonic body where Sox2 expression needs to be suppressed. Studies have reported that USP22 functions as a transcriptional repressor by occupying and deubiquitinating $\mathrm{H} 2 \mathrm{~B}$ at the Sox2-promoter region during the differentiation of ESC into the embryonic body. USP22 expression is also important for regulating neural stem/progenitor cell maintenance through the Notch signaling pathway ${ }^{[18]}$. Deubiquitination by USP22 stabilizes the expression of Hes1 protein that negatively modulates neuronal differentiation. On the contrary, depletion of USP22 delays Hes1 oscillation and thereby, induces neuronal differentiation from neuronal progenitor stem cells ${ }^{[39]}$. Overall, USP22 functions in multiple pathways to maintain cellular homeostasis and physiological functions of cells.

\section{USP22 EXPRESSION IS FREQUENTLY ALTERED IN CANCER}

Altered expression of USP22 was first detected in microarray studies from patient tissue cohorts where the expression of 11-gene signatures in stem like cells correlates with poor prognosis of the cancer ${ }^{[17]}$. Over the years, upregulation of USP22 has been validated in several cancers such as breast, colorectal, pancreatic, lung, ovarian, bladder, lymphoma, glioma, mesothelioma, neuroblastoma, etc. ${ }^{[17,31,49-53]}$. USP22 
mainly functions as a part of the SAGA complex and depletion of USP22 alters the expression of a variety of transcriptional regulators that ultimately affect the cellular conserved pathway or cell metabolism ${ }^{[54]}$. On the contrary, overexpression of USP22 often stabilizes the transcriptional effector proteins that directly or indirectly influence gene expression. Higher expression of USP22 is associated with increased risk of cancer recurrence and poor disease-free survival ${ }^{[19]}$. USP22 expression also correlates with cell cycle progression. In fact, depletion of USP22 has been shown to be associated with cell cycle arrest, mainly at the Go/G1 phase ${ }^{[18,48,55,56]}$. Moreover, the depletion of USP22 was shown to decrease in vivo tumor growth ${ }^{[1,55]}$.

The oncogenic role of USP22 in cancer stem cells (CSC) has been identified as a poor prognostic factor in multiple cancer models. Mechanistic studies indicate that by deubiquitination, USP22 has been associated with the stabilization of a variety of its downstream proteins that are important for the development and maintenance of CSC including BMI1. It has also been shown that the increased stability of BMI1 induces CSC populations by inducing the expression of stemness associated genes such as CD133, SOX2, OCT4 and NANOG and thereby, favor the progression of gastric cancer ${ }^{[57]}$. The role of USP22 and BMI 1 in glioma associated stem cells has also been reported. Under hypoxic conditions, USP22 stabilizes BMI1 to induce CSC formation for cancer progression in glioma models ${ }^{[58]}$.

In non-small cell lung cancer (NSCLC), the upregulation of USP22 was reported to be associated with advanced stage or recurrent NSCLC and considered as a poor prognostic marker for overall survival ${ }^{[59]}$. Knockdown of USP22 in an in vivo model was shown to decrease tumor angiogenesis, impair nonhomologous DNA damage repair pathways and significantly improve the therapeutic efficacy of cisplatin. USP22 upregulation affects a broad range of pathways in NSCLC to maintain tumor aggressiveness. Cisplatin-resistant lung adenocarcinoma cells were shown to be associated with upregulation of USP22. According to that model, USP22 enhances DNA damage repair and cisplatin resistance by deubiquitinating histone $\mathrm{H} 2 \mathrm{~A}$, which in turn facilitates the phosphorylation of histone $\mathrm{H} 2 \mathrm{AX}$. In addition, $\mathrm{USP} 22$ was shown to decrease the acetylation of Ku70 by stabilizing SIRT11 via deubiquitination. Ku70 acetylation dissociates the Bax-Ku70 interaction and thereby, induces apoptosis by favoring mitochondrial translocation of Bax. However, upregulation of USP22 in lung adenocarcinoma inhibits Bax-mediated apoptosis in cisplatin-resistant cells ${ }^{[52]}$. Upregulation of USP22 was also shown to be associated with chemotherapy-resistant pancreatic cancer cell survival by enhancing autophagic activity ${ }^{[60]}$. In breast and colorectal cancer, upregulation of USP22 was reported to be associated with decreased therapeutic efficacy of the HSP9o inhibitor ganetespib. Depletion of USP22 in an in vivo model of colorectal cancer was shown to increase the therapeutic potentiation of ganetespib ${ }^{[61]}$.

In gastric cancer, the co-expression of USP22 and BMI1 was shown to be associated with shorter diseasefree survival and a poor prognosis for overall survival ${ }^{[62]}$. This was similarly reported in colon cancers. The upregulated expression of USP22 was significantly correlated with both a decrease in relapse-free survival and overall survival. An in vitro study showed that the upregulation of USP22 mediated the enhanced expression of BMI1 and Cyclin D2, and was responsible for increased cell proliferation and the metastatic behavior of colon cancer cells ${ }^{[63]}$. In hepatocellular carcinoma, the enhanced expression of USP22 was shown to be an independent factor for a poor prognosis with tumor progression ${ }^{[64]}$. The enhanced stability of c-Myc following USP22 mediated deubiquitination was reported to be associated with breast cancer cell proliferation and metastatic activity ${ }^{[43]}$. The upregulation of USP22 was also reported to be associated with a poor prognosis in papillary thyroid carcinoma ${ }^{[65]}$ and glioma ${ }^{[66]}$. In retinoblastoma, the depletion of USP22 has been shown to induce cancer cell apoptosis by suppressing the TERT/P53 signal pathway ${ }^{[67]}$.

In the majority of cancers, USP22 functions like an oncogene. Tumor suppressive functions however, were also reported in certain cancer models such as acute myeloid leukemia (AML) and colorectal cancer. Recently, in an in vivo model, it was shown that the deletion of USP22 from Mx1-Cre mice carrying 
$\mathrm{Kras}^{\mathrm{G12D/+}}$ was associated with shorter survival compared to $\mathrm{Kras}^{\mathrm{G12D/+}}$ mice. Further studies indicate that mice that received myeloid progenitor cells carrying USP22 deletion and mutated $\mathrm{Kras}^{\mathrm{G}}{ }^{2 \mathrm{D} /+}$ had an AML phenotype. As a mechanism, USP22 was shown to positively regulate protein expression of the transcription factor PU.1, which is important for myeloid and B-lymphoid cell development. Depletion of USP22 directly affected myeloid specific gene expression in $\mathrm{Kras}^{\mathrm{G} 12 \mathrm{D} / \mathrm{t}}$ mutated mice, which further led to the development of $\mathrm{AML}^{[68]}$. Contradictory functions of USP22 in the development of colorectal cancer have been reported. One such study showed that intestine specific USP22 deletion impaired the tumor phenotype associated with $A p c$ mutation and positively correlated with the intestinal tumor burden and decreased survival. Mechanistically, the loss of USP22 resulted in increased mTOR activity and has been linked to the tumorigenic properties of colorectal carcinoma ${ }^{[69]}$.

Over-expression of USP22 is observed in aggressive PCa and has been associated with its oncogenic function. In the following section, we will concentrate mainly on the role of USP22 in the development of CRPC and treatment-resistant PCa.

\section{USP22 AND PROSTATE CANCER}

During PCa progression, increase in copy numbers as well as enhanced expression of androgen receptor (AR) (along with its splice variant formation) often led to aggressive therapy resistant phenotypes ${ }^{[70,7]}$. Therefore, targeting AR is the most favorable choice to limit PCa progression. Over the years, improvement in AR targeted therapy has increased overall survival to some extent, however, recent clinical studies indicate that a individuals are becoming resistant to second generation anti-androgen therapy. Therefore, understanding therapy resistance pathways may provide better or alternative solutions to target PCa. Since the 11-gene signature was shown to predict PCa recurrence and therapy resistance, the contribution of individual genes and 5-year PCa survival was analyzed in mCRPC cases. High expression of Ki-67, BUB1, KNTC1 and USP22 showed significant association with poor 5 -year survival ${ }^{[18]}$. Further, it was shown that the concerted expression of USP22, AR and Myc in PCa cells predicted the worst prognosis of the disease. USP22 plays an important role in AR protein stability and recruitment to AR-binding regions to drive AR driven cancer cell proliferation and tumor growth in CRPC cells. Later, it was shown that USP22 is equally important for protein stability of AR-variants. The upregulation of USP22 also promotes AR/ Myc driven gene expression independent of androgens in the CRPC cell line, implicating that USP22 has a tremendous impact on genes that are regulated by AR and Myc in CRPC cells. This might be important to the phenomenon of anti-androgen therapy resistance ${ }^{[18]}$. Interestingly, analysis of patient data with $\mathrm{mCRPC}$ validates that point (https://www.cbioportal.org// ${ }^{[72]}$. Analysis of the coordinated expression of USP22 and $\mathrm{AR}$ between abiraterone/enzalutamide (2nd generation anti-androgen therapy) in naïve $v s$. exposed groups revealed that USP22/AR expression is upregulated in patients who have progressed to mCRPC under treatment conditions. However, in the treatment naïve group, such correlation was not ascertained [Figure 1]. Patients who are resistant to abiraterone/enzalutamide therapy often develop neuroendocrinelike PCa. Further analysis of patient data (GSE126078) indicates that in pathologically validated neuroendocrine PCa, USP22 expression is significantly higher compared to metastatic sites, which did not develop the neuroendocrine phenotype [Figure 2]. In general, bone is the preferred metastatic site for PCa. However, neuroendocrine PCas often develop visceral metastasis. USP22 expression in visceral metastatic sites (https://www.cbioportal.org/) ${ }^{[72]}$ was significantly higher compared to bone [Figure 3]. Therefore, further validation of the earlier observations and selective upregulation of USP22 are associated with therapy resistance and progression of the disease. This group of patients need an alternative form of treatment and the early detection and stratification of these patients will be beneficial.

Increased expression of USP22 was also observed with progression of primary PCa. Analysis of the Oncomine database showed that USP22 expression increases with increased Gleason score [Figures 4 and 5] ${ }^{[73,74]}$, indicating that during progression of PCa, USP22 expression can be a predictive factor for advanced 


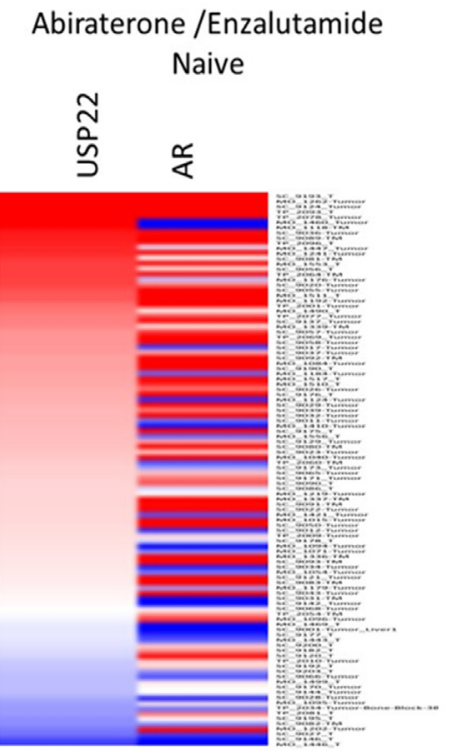

Abiraterone /Enzalutamide

$$
\text { Exposed }
$$

กิ

$\stackrel{\text { ڤ }}{\mathrm{c}}$

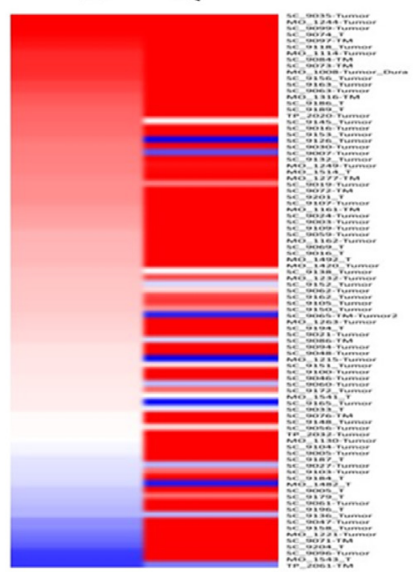

Figure 1. Analysis of ubiquitin-specific peptidase 22 (USP22) and androgen receptor (AR) expression from metastatic biopsy samples deposited in https://github.com/cBioPortal/datahub/tree/master/public/prad_su2c_2019

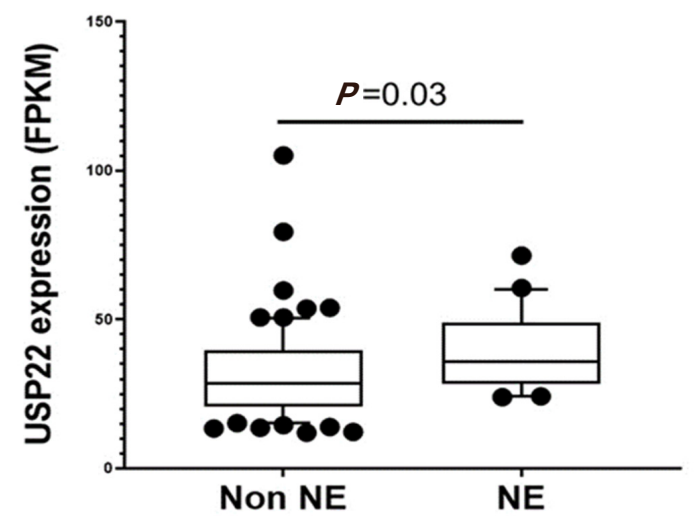

Figure 2. Ubiquitin-specific peptidase 22 (USP22) expression between neuroendocrine (NE) vs. patients who did not develop neuroendocrine PCa (Non NE) using the GSE126078 database

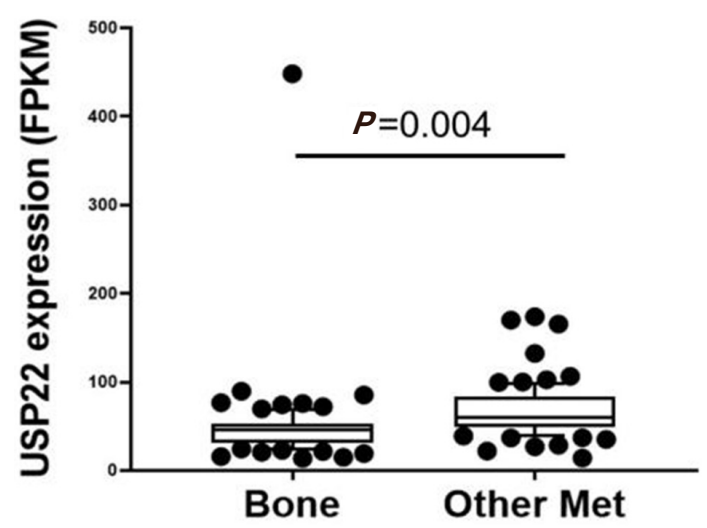

Figure 3. Ubiquitin-specific peptidase 22 (USP22) expression compared between bone and visceral metastatic sites (Other met) using the expression data deposited in cbioportal (https://www.cbioportal.org/study/summary?id=prad_su2c_2019, SU2C/PCF Dream Team, PNAS 2019) 


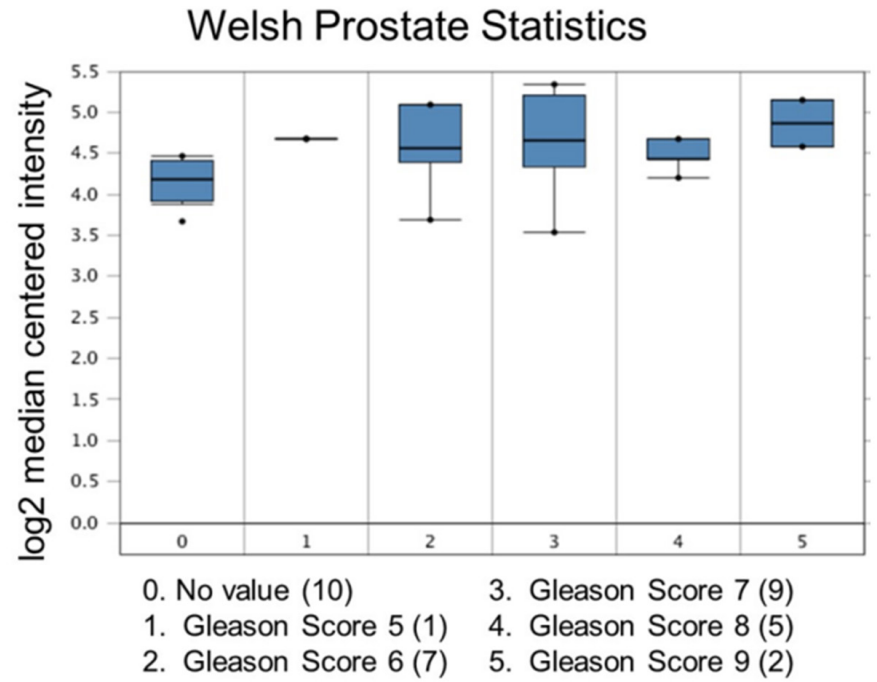

Figure 4. Ubiquitin-specific peptidase 22 expression across the Gleason Score. Number of patients are in parenthesis

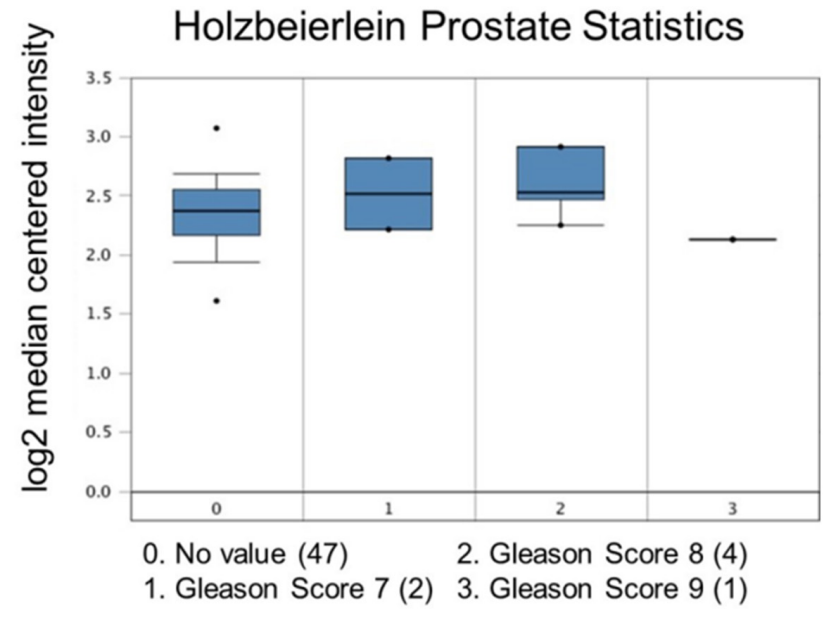

Figure 5. Ubiquitin-specific peptidase 22 expression across the Gleason Score. Number of patients are in parenthesis

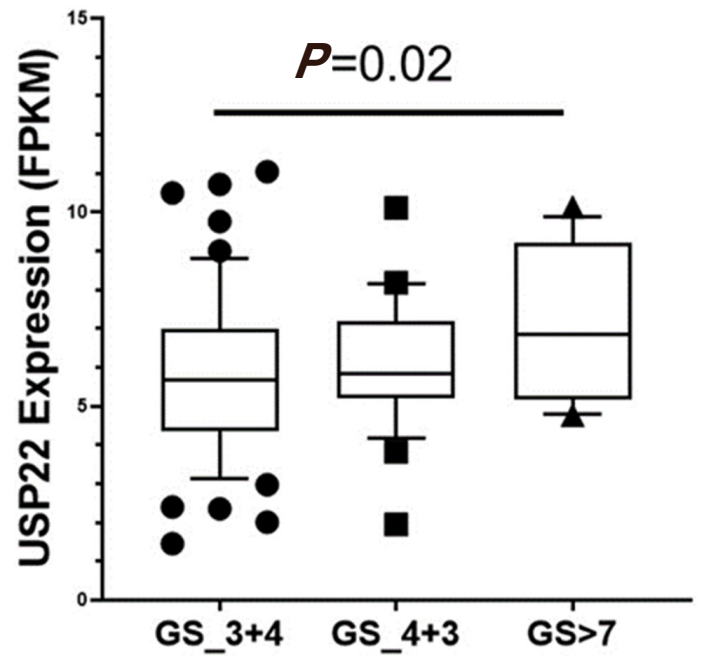

Figure 6. Ubiquitin-specific peptidase 22 expression across the Gleason Score (GS) using the database GSE54460 


\section{Grasso Prostate Statistics}

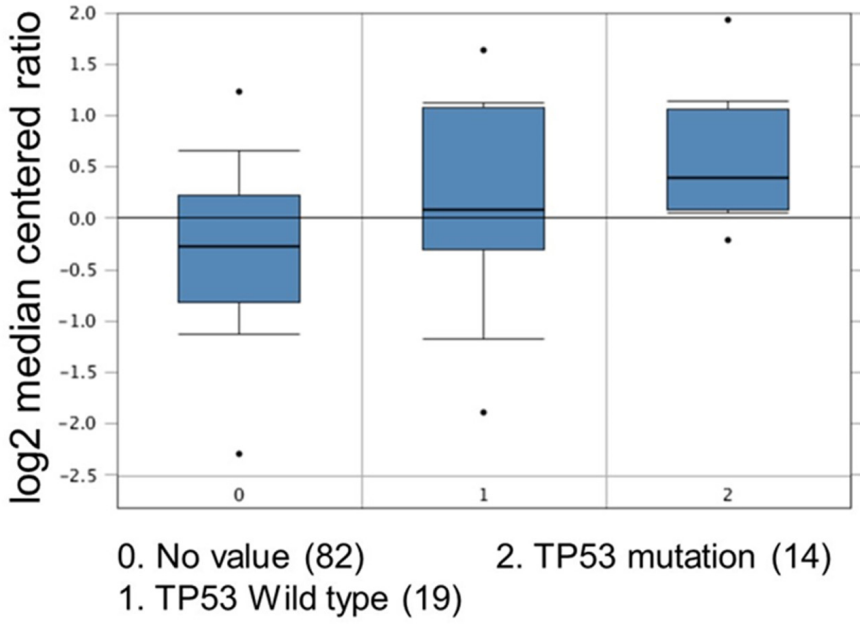

Figure 7. Comparison study for ubiquitin-specific peptidase 22 expression with TP53 mutation status. Number of patients are in parenthesis

disease. Further analysis of GSE54460 expression data supports increased USP22 expression with higher grades of PCa [Figure 6]. Moreover, advanced PCa patients often have functionally inactive TP53. Oncomine analysis of the Grasso cohort indicates that TP53 mutation is associated with increased expression of USP22 [Figure 7 $]^{[75]}$. Therefore, primary PCa patients who have higher expression of USP22 with functionally inactive TP53 might be candidates for alternative therapeutic approaches. Various clinical trials are currently ongoing with upfront administration of chemotherapy such as cabazitaxel for patients who have started to develop disease progression in the early stage. In future, analysis of such cohorts will address whether such therapy can be beneficial for those who have showed early upregulation of USP22 expression.

To mimic that hyperactivation/overexpression state, the role of USP22 functions in PCa progression has recently been redefined in a genetically modified mouse model. According to the model, prostate specific upregulation of USP22 is associated with a hyperproliferative phenotype, an indication of aberrant cell proliferation. Moreover, studies have also showed that overexpression of USP22 is important for cellular survival following a genotoxic insult by DNA-damaging agents. In line with their finding, the authors have identified the nucleotide excision repair pathway protein XPC as a substrate for USP22, which modulate XPC polyubiquitination status following the DNA-damage response and thereby, efficiently recruited it into the damage foci. Interestingly, the depletion of USP22 in PCa cells affects efficient DNA repair and therefore, presents a therapeutic challenge ${ }^{[19]}$.

Although USP22 was identified almost 15 years earlier as an important oncogenic driver for therapy resistant prostate cancer, not much work has been carried out to understand its importance in the development of mCRPC. As part of the SAGA complex, how inappropriate stoichiometric upregulation of USP22 in PCa drives AR/Myc mediated gene-expression remains unresolved. Also, whether USP22 plays an independent role in PCa progression is not well understood.

\section{OTHER USPS IN PROSTATE CANCERS}

Importantly, other ubiquitin specific proteases or USPs have long been recognized in the progression of advanced PCa.

USP2a (also known as USP2) has been associated with PCa development. More than 50\% of cases with PCa have USP2a overexpression. Increase in USP2a selectively deubiquitinates and stabilizes MDM2, which 
is important for the proteasomal degradation of p53 in PCa cells. p53 is the negative regulator of Myc in many cases. USP2a mediated enhanced stability of MDM2 abrogates p53 accumulation and its tumor suppressive functions. Therefore, the inhibition of p53 mediated transactivation of transcriptional activity indirectly stabilizes Myc accumulation in cells and thereby, enhances the development of aggressive PCa transformation. The deubiquitination activity of USP2a was also found to stabilize the anti-apoptotic gene fatty acid synthase and thereby induce cells to develop neoplastic transformation. The depletion of USP2a has also been shown to abrogate such cellular transformation ${ }^{[76,7]]}$.

USP7 has been associated with PCa and plays a negative role for PTEN nuclear localization. PTEN is generally regarded as a protein phosphatase that dephosphorylates the phosphatidylinositol $(3,4,5)$-triphosphate to inhibit AKT signaling. However, PTEN's role in the nuclear DNA repair system associated with tumor suppressive functions has been well recognized. Following mono-ubiquitination, PTEN moves into the nucleus and participates in the repair processes. In PCa, over-expression of USP7 expels this ubiquitinated-PTEN to the cytosol and activates the cells towards transformation. Interestingly, in the presence of androgen, USP7 was identified as a co-regulator of AR. Studies also suggest that USP7 mediated AR-deubiquitination enhance the AR-transcriptional ability that promotes cell proliferation and PCa aggressiveness. Moreover, single nucleotide polymorphisms that affect USP7 function has been associated with the development of intermediate risk PCas ${ }^{[78,79]}$.

USP19 silencing directly affects the growth of several prostate cancer cell lines, suggesting a putative role in carcinogenesis $^{[80]}$. USP19 deubiquitinates and stabilizes KPC1, an E3 ligase for p27. Interestingly, the effects of decreased nuclear levels of p27, resulting in a poor prognosis, have already been described in prostate cancer $^{[81]}$. USP19 regulates the levels of p27, although p27 is not a USP19 substrate. Reports indicate that the disruption of USP19 inhibits a series of PCa cell proliferation by arresting cells in the G1 to S phase transition through stabilization of the cyclin-dependent kinase inhibitor $\mathrm{p} 27^{[80]}$. Increased stability of AR by USP12, USP14 and USP26 has been linked to the development of aggressive $\mathrm{PCa}^{[82-84]}$. Recent reports indicate that the overexpression of USP33 in PCa confers docetaxel resistance by inhibiting JNK activation and apoptosis ${ }^{[85]}$.

In the context of PCa, most USPs are overexpressed; however, USP9x was found to be down regulated in advanced PCa and was associated with higher Gleason scores. This downregulation increases the local invasiveness of PCa cells, possibly through the ERK activation pathway ${ }^{[8]}$.

Among all the DUBs, available data suggest that USP22 functions often overlapped with other reported USPs in the context of progression and development of therapy resistant PCas. Therefore, USP22 targeted therapy or broad-spectrum inhibitors that can abrogate the functions of a group of USPs may be a better therapeutic agent in PCa.

\section{TARGETING USP22 IN ADVANCED PROSTATE CANCER TREATMENT}

Recent studies have suggested that USP22 is emerging as a potential oncogenic driver in relation to PCa. As a member of the cysteine protease family, its catalytic domains are somewhat conserved amongst family members. Therefore, the development of inhibitors specifically against one such member is challenging. Efforts have been made to develop small molecule inhibitors against the allosteric sites of USP22. However, till now, no such specific inhibitor has been validated to target USP22. Recently, Pirarubicin (4'-O-tetrahydropyranyl doxorubicin, THP), an anthracycline (analogue of another chemotherapeutic agent known as doxorubicin), has been shown to inhibit USP22 expression in a condition-specific manner ${ }^{[87]}$. Reports indicated that protein kinase A (PKA), protein kinase B or mitogen activated kinase-mediated phosphorylation of CREB-1 bind and activate the USP22 promoter for its synthesis. The addition of THP abrogates PKA activity and decreases CREB-1 phosphorylation,, thereby inhibiting USP22 expression and 


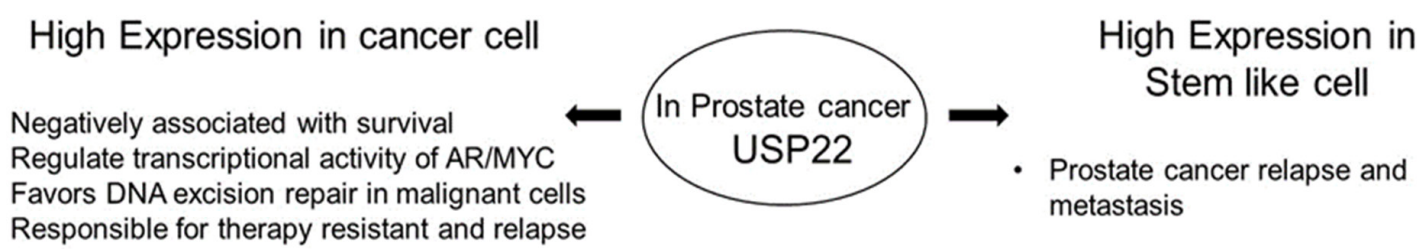

Figure 8. Summary of ubiquitin-specific peptidase 22 (USP22) role in prostate cancer

USP22 mediated tumorigenic activity. Betulinic acid (BA), a small molecule isolated from white birch trees, has been shown to inhibit an array of DUBs. BA was also showed to reduce AR protein stability and selectively kills PCa cells. Another multi-DUB inhibitor WP1130 has been shown to selectively kill PCa cells. Treatment with WP1130 also reduces AR expression in CRPC cells. Therefore, BA and WP1130 have the potential to enhance the therapeutic efficacy of CRPC cells and the published literature suggests that the combination of these inhibitors with enzalutamide increases the therapeutic window for the treatment of advanced PCa patients ${ }^{[88]}$. With such growing knowledge, scientists have tried to develop exosite inhibitors against the various DUBs. One such inhibitor, P5091, is highly selective against USP7 and has been shown to induce apoptotic cell death in therapy resistant multiple myeloma cells ${ }^{[89]}$. However, its selectivity and specificity as an agent in PCa remains unknown.

\section{CONCLUSION}

In summary, the oncogenic role of upregulated USP22 in the progression and development of treatment resistance of PCas has been observed [Figure 8] ${ }^{[19]}$. Accumulated evidence indicates that USP22 possibly functions independent of the SAGA complex in the progression of PCas. Increased acetylation and enhanced activity of GCN5 has been reported to be associated with advanced PCa. However, there is a lack of studies to ascertain any relationship between upregulated USP22 and other members of the SAGA complex in the development of aggressive PCas. Moreover, in advanced PCas, the coordinated function of upregulated Myc and USP22 indicates the lack of feedback regulation by hyperactivated Myc. Therefore, to develop better targeted therapeutic approaches, a comprehensive understanding about the functional interactions among the various sub-units of SAGA and their relationships with AR/Myc is important. Moreover, the differential functions of USP22 in the normal prostate, aggressive disease and disease progression are not fully understood. Thus, defining the role of USP22 will be beneficial for the development of future therapeutic modalities.

\section{DECLARATIONS}

\section{Authors' contributions}

Wrote the paper jointly and SD analyze the data: Nag N, Dutta S

\section{Availability of data and materials}

Not applicable.

\section{Financial support and sponsorship}

This work was supported by grants (1R21CA241234-01) to Dutta S.

\section{Conflicts of interest}

Both authors declared that there are no conflicts of interest.

\section{Ethical approval and consent to participate}

Not applicable. 


\section{Consent for publication}

Not applicable.

\section{Copyright}

(c) The Author(s) 2020.

\section{REFERENCES}

1. Siegel RL, Jemal A, Wender RC, Gansler T, Ma J, et al. An assessment of progress in cancer control. CA Cancer J Clin 2018;68:329-39.

2. Rathkopf DE, Antonarakis ES, Shore ND, Tutrone RF, Alumkal JJ, et al. Safety and antitumor activity of apalutamide (ARN-509) in metastatic castration-resistant prostate cancer with and without prior abiraterone acetate and prednisone. Clin Cancer Res 2017;23:354451.

3. Rathkopf DE, Smith MR, Ryan CJ, Berry WR, Shore ND, et al. Androgen receptor mutations in patients with castration-resistant prostate cancer treated with apalutamide. Ann Oncol 2017;28:2264-71.

4. Scher HI, Fizazi K, Saad F, Taplin ME, Sternberg CN, et al. Increased survival with enzalutamide in prostate cancer after chemotherapy. N Engl J Med 2012;367:1187-97.

5. Zhou CK, Check DP, Lortet-Tieulent J, Laversanne M, Jemal A, et al. Prostate cancer incidence in 43 populations worldwide: an analysis of time trends overall and by age group. Int J Cancer 2016;138:1388-400.

6. Berish RB, Ali AN, Telmer PG, Ronald JA, Leong HS. Translational models of prostate cancer bone metastasis. Nat Rev Urol 2018;15:403-21.

7. Clarke NW, Hart CA, Brown MD. Molecular mechanisms of metastasis in prostate cancer. Asian J Androl 2009;11:57-67.

8. Yoshida T, Kinoshita H, Taniguchi H, Yanishi M, Sugi M, et al. A randomized, open-label, controlled trial of monthly oral minodronate or semiannual subcutaneous injection of denosumab for bone loss by androgen deprivation in Asian men with prostate cancer: the prevention of osteopenia with minodronate and denosumab (PROMADE) study. Osteoporos Int 2020;31:1251-9.

9. Deantoni CL, Fodor A, Cozzarini C, Fiorino C, Brombin C, et al. Prostate cancer with low burden skeletal disease at diagnosis: outcome of concomitant radiotherapy on primary tumor and metastases. Br J Radiol 2020;93:20190353.

10. DiNatale A, Fatatis A. The bone microenvironment in prostate cancer metastasis. Adv Exp Med Biol 2019;1210:171-84

11. Wang L, Xu M, Kao CY, Tsai SY, Tsai MJ. Small molecule JQ1 promotes prostate cancer invasion via BET-independent inactivation of FOXA1. J Clin Invest 2020;130:1782-92.

12. Ku SY, Rosario S, Wang Y, Mu P, Seshadri M, et al. Rb1 and Trp53 cooperate to suppress prostate cancer lineage plasticity, metastasis, and antiandrogen resistance. Science 2017;355:78-83.

13. Mu P, Zhang Z, Benelli M, Karthaus WR, Hoover E, et al. SOX2 promotes lineage plasticity and antiandrogen resistance in TP53- and RB1-deficient prostate cancer. Science 2017;355:84-8.

14. Jaratlerdsiri W, Chan EKF, Gong T, Petersen DC, Kalsbeek AMF, et al. Whole-genome sequencing reveals elevated tumor mutational burden and initiating driver mutations in african men with treatment-naive, high-risk prostate cancer. Cancer Res 2018;78:6736-46.

15. Zolotovskaia MA, Sorokin MI, Petrov IV, Poddubskaya EV, Moiseev AA, et al. Disparity between inter-patient molecular heterogeneity and repertoires of target drugs used for different types of cancer in clinical oncology. Int J Mol Sci 2020;21.

16. Su X, Long Q, Bo J, Shi Y, Zhao LN, et al. Mutational and transcriptomic landscapes of a rare human prostate basal cell carcinoma. Prostate 2020;80:508-17.

17. Glinsky GV, Berezovska O, Glinskii AB. Microarray analysis identifies a death-from-cancer signature predicting therapy failure in patients with multiple types of cancer. J Clin Invest 2005;115:1503-21.

18. Schrecengost RS, Dean JL, Goodwin JF, Schiewer MJ, Urban MW, et al. USP22 regulates oncogenic signaling pathways to drive lethal cancer progression. Cancer Res 2014;74:272-86.

19. McCann JJ, Vasilevskaya IA, Poudel Neupane N, Shafi AA, McNair C, et al. USP22 functions as an oncogenic driver in prostate cancer by regulating cell proliferation and DNA Repair. Cancer Res 2020;80:430-43.

20. Pfoh R, Lacdao IK, Georges AA, Capar A, Zheng H, et al. Crystal structure of USP7 ubiquitin-like domains with an ICP0 peptide reveals a novel mechanism used by viral and cellular proteins to target USP7. PLoS Pathog 2015;11:e1004950.

21. Reyes-Turcu FE, Ventii KH, Wilkinson KD. Regulation and cellular roles of ubiquitin-specific deubiquitinating enzymes. Annu Rev Biochem 2009; 78:363-97.

22. Sahtoe DD, Sixma TK. Layers of DUB regulation. Trends Biochem Sci 2015;40:456-67.

23. Espinosa JM. Histone H2B ubiquitination: the cancer connection. Genes Dev 2008;22:2743-9.

24. Poondla N, Chandrasekaran AP, Kim KS, Ramakrishna S. Deubiquitinating enzymes as cancer biomarkers: new therapeutic opportunities? BMB Rep 2019;52:181-9.

25. Melo-Cardenas J, Zhang Y, Zhang DD, Fang D. Ubiquitin-specific peptidase 22 functions and its involvement in disease. Oncotarget 2016;7:44848-56.

26. Fraile JM, Quesada V, Rodriguez D, Freije JM, Lopez-Otin C. Deubiquitinases in cancer: new functions and therapeutic options. Oncogene 2012;31:2373-88.

27. Atanassov BS, Evrard YA, Multani AS, Zhang Z, Tora L, et al. Gen5 and SAGA regulate shelterin protein turnover and telomere maintenance. Mol Cell 2009;35:352-64. 
28. Ramachandran S, Haddad D, Li C, Le MX, Ling AK, et al. The SAGA Deubiquitination Module Promotes DNA Repair and Class Switch Recombination through ATM and DNAPK-Mediated gammaH2AX Formation. Cell Rep 2016;15:1554-65.

29. Armour SM, Bennett EJ, Braun CR, Zhang XY, McMahon SB, et al. A high-confidence interaction map identifies SIRT1 as a mediator of acetylation of USP22 and the SAGA coactivator complex. Mol Cell Biol 2013;33:1487-502.

30. Young MJ, Hsu KC, Lin TE, Chang WC, Hung JJ. The role of ubiquitin-specific peptidases in cancer progression. J Biomed Sci 2019;26:42.

31. Zhang Y, Yao L, Zhang X, Ji H, Wang L, et al. Elevated expression of USP22 in correlation with poor prognosis in patients with invasive breast cancer. J Cancer Res Clin Oncol 2011;137:1245-53.

32. Daniel JA, Grant PA. Multi-tasking on chromatin with the SAGA coactivator complexes. Mutat Res 2007;618:135-48.

33. Koehler C, Bonnet J, Stierle M, Romier C, Devys D, et al. DNA binding by Sgf11 protein affects histone H2B deubiquitination by SptAda-Gcn5-acetyltransferase (SAGA). J Biol Chem 2014;289:8989-99.

34. Henry KW, Wyce A, Lo WS, Duggan LJ, Emre NC, et al. Transcriptional activation via sequential histone H2B ubiquitylation and deubiquitylation, mediated by SAGA-associated Ubp8. Genes Dev 2003;17:2648-63.

35. Henry KW, Berger SL. Trans-tail histone modifications: wedge or bridge? Nat Struct Biol 2002;9:565-6.

36. Zhao Y, Lang G, Ito S, Bonnet J, Metzger E, et al. A TFTC/STAGA module mediates histone H2A and H2B deubiquitination, coactivates nuclear receptors, and counteracts heterochromatin silencing. Mol Cell 2008;29:92-101.

37. Ao N, Liu Y, Feng H, Bian X, Li Z, et al. Ubiquitin-specific peptidase USP22 negatively regulates the STAT signaling pathway by deubiquitinating SIRT1. Cell Physiol Biochem 2014;33:1863-75.

38. Lin Z, Yang H, Kong Q, Li J, Lee SM, et al. USP22 antagonizes p53 transcriptional activation by deubiquitinating Sirt1 to suppress cell apoptosis and is required for mouse embryonic development. Mol Cell 2012;46:484-94.

39. Kobayashi T, Iwamoto Y, Takashima K, Isomura A, Kosodo Y, et al. Deubiquitinating enzymes regulate Hes1 stability and neuronal differentiation. FEBS J 2015;282:2411-23.

40. Gao Y, Lin F, Xu P, Nie J, Chen Z, et al. USP22 is a positive regulator of NFATc2 on promoting IL2 expression. FEBS Lett 2014;588:87883.

41. Xiao H, Tian Y, Yang Y, Hu F, Xie X, et al. USP22 acts as an oncogene by regulating the stability of cyclooxygenase-2 in non-small cell lung cancer. Biochem Biophys Res Commun 2015;460:703-8.

42. Wilson MA, Koutelou E, Hirsch C, Akdemir K, Schibler A, et al. Ubp8 and SAGA regulate Snfl AMP kinase activity. Mol Cell Biol 2011;31:3126-35.

43. Kim D, Hong A, Park HI, Shin WH, Yoo L, et al. Deubiquitinating enzyme USP22 positively regulates c-Myc stability and tumorigenic activity in mammalian and breast cancer cells. J Cell Physiol 2017;232:3664-76.

44. Atanassov BS, Dent SY. USP22 regulates cell proliferation by deubiquitinating the transcriptional regulator FBP1. EMBO Rep 2011;12:924-30.

45. Li C, Irrazabal T, So CC, Berru M, Du L, et al. The H2B deubiquitinase Usp22 promotes antibody class switch recombination by facilitating non-homologous end joining. Nat Commun 2018;9:1006.

46. Xiong J, Che X, Li X, Yu H, Gong Z, et al. Cloning and characterization of the human USP22 gene promoter. PLoS One 2012;7:e52716.

47. Xiong J, Zhou X, Gong Z, Wang T, Zhang C, et al. PKA/CREB regulates the constitutive promoter activity of the USP22 gene. Oncol Rep 2015;33:1505-11.

48. Gennaro VJ, Stanek TJ, Peck AR, Sun Y, Wang F, et al. Control of CCND1 ubiquitylation by the catalytic SAGA subunit USP22 is essential for cell cycle progression through G1 in cancer cells. Proc Natl Acad Sci U S A 2018;115:E9298-307.

49. Piao S, Liu Y, Hu J, Guo F, Ma J, et al. USP22 is useful as a novel molecular marker for predicting disease progression and patient prognosis of oral squamous cell carcinoma. PLoS One 2012;7:e42540.

50. Hu J, Liu YL, Piao SL, Yang DD, Yang YM, et al. Expression patterns of USP22 and potential targets BMI-1, PTEN, p-AKT in nonsmall-cell lung cancer. Lung Cancer 2012;77:593-9.

51. Yang M, Liu YD, Wang YY, Liu TB, Ge TT, et al. Ubiquitin-specific protease 22: a novel molecular biomarker in cervical cancer prognosis and therapeutics. Tumour Biol 2014;35:929-34.

52. Wang A, Ning Z, Lu C, Gao W, Liang J, et al. USP22 induces cisplatin resistance in lung adenocarcinoma by regulating gammaH2AXmediated DNA damage repair and Ku70/Bax-mediated apoptosis. Front Pharmacol 2017;8:274.

53. Liu YL, Yang YM, Xu H, Dong XS. Aberrant expression of USP22 is associated with liver metastasis and poor prognosis of colorectal cancer. J Surg Oncol 2011;103:283-9.

54. Lang G, Bonnet J, Umlauf D, Karmodiya K, Koffler J, et al. The tightly controlled deubiquitination activity of the human SAGA complex differentially modifies distinct gene regulatory elements. Mol Cell Biol 2011;31:3734-44.

55. Zhang K, Yang L, Wang J, Sun T, Guo Y, et al. Ubiquitin-specific protease 22 is critical to in vivo angiogenesis, growth and metastasis of non-small cell lung cancer. Cell Commun Signal 2019;17:167.

56. Lin Z, Tan C, Qiu Q, Kong S, Yang H, et al. Ubiquitin-specific protease 22 is a deubiquitinase of CCNB1. Cell Discov $2015 ; 1$.

57. Ma Y, Fu HL, Wang Z, Huang H, Ni J, et al. USP22 maintains gastric cancer stem cell stemness and promotes gastric cancer progression by stabilizing BMI1 protein. Oncotarget 2017;8:33329-42.

58. Qiu GZ, Liu Q, Wang XG, Xu GZ, Zhao T, et al. Hypoxia-induced USP22-BMI1 axis promotes the stemness and malignancy of glioma stem cells via regulation of HIF-1alpha. Life Sci 2020;247:117438.

59. Ning J, Zhang J, Liu W, Lang Y, Xue Y, et al. Overexpression of ubiquitin-specific protease 22 predicts poor survival in patients with early-stage non-small cell lung cancer. Eur J Histochem 2012;56:e46. 
60. Liang JX, Ning Z, Gao W, Ling J, Wang AM, et al. Ubiquitinspecific protease 22induced autophagy is correlated with poor prognosis of pancreatic cancer. Oncol Rep 2014;32:2726-34.

61. Kosinsky RL, Helms M, Zerche M, Wohn L, Dyas A, et al. USP22-dependent HSP90AB1 expression promotes resistance to HSP90 inhibition in mammary and colorectal cancer. Cell Death Dis 2019;10:911.

62. Yang DD, Cui BB, Sun LY, Zheng HQ, Huang Q, et al. The co-expression of USP22 and BMI-1 may promote cancer progression and predict therapy failure in gastric carcinoma. Cell Biochem Biophys 2011;61:703-10.

63. Yuan X, Wang H, Xu A, Zhu X, Zhan Y, et al. Ubiquitin-specific peptidase 22 promotes proliferation and metastasis in human colon cancer. Oncol Lett 2019;18:5567-76.

64. Tang B, Tang F, Li B, Yuan S, Xu Q, et al. High USP22 expression indicates poor prognosis in hepatocellular carcinoma. Oncotarget 2015;6:12654-67.

65. Wang H, Li YP, Chen JH, Yuan SF, Wang L, et al. Prognostic significance of USP22 as an oncogene in papillary thyroid carcinoma. Tumour Biol 2013;34:1635-9.

66. Hong A, Lee JE, Chung KC. Ubiquitin-specific protease 22 (USP22) positively regulates RCAN1 protein levels through RCAN1 deubiquitination. J Cell Physiol 2015;230:1651-60.

67. Zhou D, Liu P, Sun DW, Chen ZJ, Hu J, et al. USP22 down-regulation facilitates human retinoblastoma cell aging and apoptosis via inhibiting TERT/P53 pathway. Eur Rev Med Pharmacol Sci 2017;21:2785-92.

68. Melo-Cardenas J, Xu Y, Wei J, Tan C, Kong S, et al. USP22 deficiency leads to myeloid leukemia upon oncogenic Kras activation through a PU.1-dependent mechanism. Blood 2018;132:423-34.

69. Kosinsky RL, Zerche M, Saul D, Wang X, Wohn L, et al. USP22 exerts tumor-suppressive functions in colorectal cancer by decreasing mTOR activity. Cell Death Differ 2020;27:1328-40.

70. Cato L, de Tribolet-Hardy J, Lee I, Rottenberg JT, Coleman I, et al. ARv7 represses tumor-suppressor genes in castration-resistant prostate cancer. Cancer Cell 2019;35:401-13.e6.

71. Sharp A, Coleman I, Yuan W, Sprenger C, Dolling D, et al. Androgen receptor splice variant-7 expression emerges with castration resistance in prostate cancer. J Clin Invest 2019;129:192-208.

72. Abida W, Cyrta J, Heller G, Prandi D, Armenia J, et al. Genomic correlates of clinical outcome in advanced prostate cancer. Proc Nat Acad Sci U S A 2019;116:11428-36.

73. Welsh JB, Sapinoso LM, Su AI, Kern SG, Wang-Rodriguez J, et al. Analysis of gene expression identifies candidate markers and pharmacological targets in prostate cancer. Cancer Res 2001;61:5974-8.

74. Holzbeierlein J, Lal P, LaTulippe E, Smith A, Satagopan J, et al. Gene expression analysis of human prostate carcinoma during hormonal therapy identifies androgen-responsive genes and mechanisms of therapy resistance. Am J Pathol 2004;164:217-27.

75. Grasso CS, Wu YM, Robinson DR, Cao X, Dhanasekaran SM, et al. The mutational landscape of lethal castration-resistant prostate cancer. Nature 2012;487:239-43.

76. Nelson WG, De Marzo AM, Yegnasubramanian S. USP2a activation of MYC in prostate cancer. Cancer Discov 2012;2:206-7.

77. Priolo C, Tang D, Brahamandan M, Benassi B, Sicinska E, et al. The isopeptidase USP2a protects human prostate cancer from apoptosis. Cancer Res 2006;66:8625-32.

78. Morra F, Merolla F, Napolitano V, Ilardi G, Miro C, et al. The combined effect of USP7 inhibitors and PARP inhibitors in hormonesensitive and castration-resistant prostate cancer cells. Oncotarget 2017;8:31815-29.

79. Wang Z, Kang W, You Y, Pang J, Ren H, et al. USP7: novel drug target in cancer therapy. Front Pharmacol 2019;10:427.

80. Lu Y, Bedard N, Chevalier S, Wing SS. Identification of distinctive patterns of USP19-mediated growth regulation in normal and malignant cells. PLoS One 2011;6:e15936.

81. Chu IM, Hengst L, Slingerland JM. The Cdk inhibitor p27 in human cancer: prognostic potential and relevance to anticancer therapy. Nat Rev Cancer 2008;8:253-67.

82. McClurg UL, Azizyan M, Dransfield DT, Namdev N, Chit N, et al. The novel anti-androgen candidate galeterone targets deubiquitinating enzymes, USP12 and USP46, to control prostate cancer growth and survival. Oncotarget 2018;9:24992-5007.

83. Geng L, Chen X, Zhang M, Luo Z. Ubiquitin-specific protease 14 promotes prostate cancer progression through deubiquitinating the transcriptional factor ATF2. Biochem Biophys Res Commun 2020;524:16-21.

84. Dirac AM, Bernards R. The deubiquitinating enzyme USP26 is a regulator of androgen receptor signaling. Mol Cancer Res 2010;8:84454.

85. Guo F, Zhang C, Wang F, Zhang W, Shi X, et al. Deubiquitinating enzyme USP33 restrains docetaxel-induced apoptosis via stabilising the phosphatase DUSP1 in prostate cancer. Cell Death Differ 2019; doi: 10.1038/s41418-019-0473-8.

86. Zhang J, Wang J, Luan T, Zuo Y, Chen J, et al. Deubiquitinase USP9X regulates the invasion of prostate cancer cells by regulating the ERK pathway and mitochondrial dynamics. Oncol Rep 2019;41:3292-304.

87. Zhou X, Gan L, Liu J, Xie X, Wang T, et al. Pirarubicin reduces USP22 expression by inhibiting CREB-1 phosphorylation in HeLa cells. Exp Ther Med 2019;17:4230-6.

88. de Las Pozas A, Reiner T, De Cesare V, Trost M, Perez-Stable C. Inhibiting Multiple Deubiquitinases To Reduce Androgen Receptor Expression In Prostate Cancer Cells. Sci Rep 2018;8:13146.

89. Chauhan D, Tian Z, Nicholson B, Kumar KG, Zhou B, et al. A small molecule inhibitor of ubiquitin-specific protease-7 induces apoptosis in multiple myeloma cells and overcomes bortezomib resistance. Cancer Cell 2012;22:345-58. 